\title{
Convergent evolution of phytopathogenic pseudomonads onto hazelnut
}

\author{
Pauline W. Wang, ${ }^{1}$ Robyn L. Morgan, ${ }^{1}$ Marco Scortichini ${ }^{2}$ \\ and David S. Guttman ${ }^{1,3}$ \\ ${ }^{1}$ Department of Cell and Systems Biology, University of Toronto, Toronto, Canada \\ ${ }^{2} \mathrm{CRA}$ Istituto Sperimentale per la Frutticoltura, Via di Fioranello 52, Roma, Italy \\ ${ }^{3}$ University of Toronto Centre for the Analysis of Genome Evolution and Function, Toronto, \\ Ontario M5S 3B2, Canada
}

Correspondence

David S. Guttman

david.guttman@utoronto.ca

Received 18 August 2006

Revised 6 March 2007

Accepted 8 March 2007
Pseudomonas syringae pv. avellanae (synonym: $P$. avellanae, Pav) is the causal agent of hazelnut decline in Greece and Italy. The population structure and evolutionary relationships of 22 strains from these two countries were examined by multilocus sequence typing (MLST) of four housekeeping genes ( $g a p A, g l t A, g y r B$ and $r p o D)$. Neighbour-joining and maximum-likelihood phylogenetic analysis revealed that Greek strains isolated from the original 1976 outbreak of hazelnut decline through 1990 were very similar to Italian strains isolated from 2002 through 2004. Other Italian strains that were isolated during the 1990 s were very homogeneous and clustered in a clade that was quite distinct from the Greek isolates and Italian isolates from the 2000s. A split decomposition analysis found evidence for recombination between these two highly divergent clades in two of the four MLST housekeeping genes. Incorporating these data into a broad MLST analysis of the $P$. syringae species complex showed that the Pav Greek and Italian strains from the 2000 s clustered with $P$. syringae phylogroup 1 , which is predominantly composed of pathogens of tomato and Brassicaceae hosts, while the Pav Italian strains from the 1990s clustered in P. syringae phylogroup 2 and are most closely related to pea (Pisum sativum L.) pathogens. These results clearly indicate that the ability to infect hazelnuts has arisen twice. This evolutionary process may be due to de novo adaptation to hazelnut by local $P$. syringae strains (such as the colonizers of Leguminosae crops), or the result of genetic exchange from the original Greek Pav clonal group into a phylogroup 2 strain. The latter explanation is intriguing since there is no exchange of hazelnut propagative material between Italy and Greece, which would be a likely vector for the movement of these pathogens.

\section{INTRODUCTION}

Pseudomonas syringae pv. avellanae (synonym: P. avellanae, Pav) is the causal agent of hazelnut (Corylus avellana L.) decline in northern Greece and central Italy (Psallidas \& Panagopoulos, 1979; Scortichini, 2002). Disease symptoms include rapid wilting of the branches and trees, which can be observed from spring to autumn. In some circumstances, longitudinal cankers are noticed along the trunk. The disease was first observed in Greece in 1976 on young hazelnut plantations (Psallidas \& Panagopoulos, 1979), and within a few years plantings of the Turkish cultivar Palaz were almost completely destroyed by the disease. Similar disease foci occurred in Italy, and as in Greece, the disease first appeared in areas that were new to hazelnut cultivation (Scortichini, 2002). Hazelnut cultivars utilized in

Abbreviations: Pav, Pseudomonas syringae pv. avellanae; MLST, multilocus sequence typing.
Greece and Italy are different. The cultivar Palaz was introduced from Turkey to Greece in the late 1960s, and there are no reported cases of decline in Turkey despite the extensive cultivation of hazelnut. The cultivars Tonda Gentile Romana and Nocchione have been grown in Italy for millennia and are locally adapted, while the cultivar Palaz has not been propagated in this locale.

Genetic and phenotypic analyses have often resulted in different and even contradictory conclusions regarding the relationship between the Pav isolates from Greece and Italy. Techniques aimed at species-level discrimination revealed no differences between these two lineages. For example, amplified 16S rDNA restriction analysis (ARDRA) performed with nine restriction endonucleases was not able to discriminate between Greek and Italian Pav strains (Scortichini et al., 2002), and 16S rDNA sequencing of strains from both countries revealed an average similarity of $99.4 \%$ between the two groups (Scortichini et al., 2005). 
On the other hand, evidence of differentiation between strains from the two geographical locales has been found using repetitive-sequence PCR (Scortichini et al., 1998; Scortichini, 2002), and the analysis of plasmid profiles, which found different numbers and sizes of the plasmids (Janse et al., 1996). Multilocus enzyme electrophoresis (MLEE) reveals that Pav is strongly clonal, and shows that the Greek strains are distinct from the Italian strains (Scortichini et al., 2003).

Phenotypic analyses have identified clear differences between Greek and Italian isolates. All Greek isolates produce a fluorescent pigment under UV on King's B medium (King et al., 1954), whereas it is quite faint for Italian isolates, and it disappears after transfers on nutrient sucrose agar (Scortichini \& Angelucci, 1999). Serotyping of Pav using O-antigen monoclonal antibodies clearly differentiated Greek isolates from those obtained in Italy (Ovod et al., 1999). Nevertheless, virulence on hazelnut is similar for both groups (Scortichini et al., 2002).

The phenotypic and genetic differences observed between Pav strains from Greece and Italy, and the diverse history of hazelnut cultivation in the two countries, leads to the hypothesis of two separate origins for hazelnut decline. We tested this hypothesis using multilocus sequence typing (MLST) of 22 strains isolated in Greece, and during two epidemics in Italy. The very high resolution of this method (Hwang et al., 2005; Sarkar \& Guttman, 2004) sheds new light on the evolutionary origins of the strains responsible for these disease outbreaks, and the relationship between Pav and the rest of the P. syringae species complex.

\section{METHODS}

Bacterial strains. The 22 strains used in this study are listed in Table 1. All of these strains have been previously described by Scortichini et al. (2002, 2003). Strains were propagated in the laboratory on nutrient agar (Oxoid) with $5 \%$ sucrose, or King's B medium (King et al., 1954) at $25^{\circ} \mathrm{C}$. All data collection and subsequent analyses were performed blinded with respect to the identity or origin of the strains.

MLST. The MLST protocol was originally described by Sarkar \& Guttman (2004) with minor modification by Hwang et al. (2005). Partial sequences from four housekeeping genes were obtained: $r p o D$ encoding sigma factor 70, gyrB encoding DNA gyrase B, gltA (also known as cts) encoding citrate synthase, and gapA encoding glyceraldehyde-3-phosphate dehydrogenase (Hwang et al., 2005) (Table 2). These loci are a subset of the seven used in the original P. syringae MLST paper (Sarkar \& Guttman, 2004); they were chosen because they consistently provide robust data, and their combined level of polymorphism is sufficient to reliably resolve evolutionary relationships. Primer sequences for these loci are available in Hwang et al. (2005). Sequences from each locus were aligned using CLUSTALW (Chenna et al., 2003), and were trimmed to their minimal shared length in GeneDoc (http://www.nrbsc.org/gfx/genedoc/).

Data analysis. Neighbour-joining and maximum-likelihood phylogenetic analyses were performed on the individual and combined datasets using MEGA3 (Kumar et al., 2004) and PHYLIP version 3.6.2 (Felsenstein, 1993) and PAUP* ver4.0b10 for UNIX (Swofford, 1993). A Kimura two-parameter evolution model was employed with gamma
Table 1. Pav strains used in this study

\begin{tabular}{|llc|}
\hline Strain & \multicolumn{1}{c|}{ Origin } & Year of isolation \\
\hline BPIC 631 & Greece/Drama & 1976 \\
BPIC 641 & Greece/Kilkis & 1976 \\
BPIC 665 & Greece/Kilkis & 1976 \\
BPIC 710 & Greece/Drama & 1987 \\
BPIC 714 & Greece/Kavala & 1987 \\
BPIC 715 & Greece/Kavala & 1987 \\
BPIC 1077 & Greece/Kilkis & 1987 \\
BPIC 1422 & Greece/Kilkis & 1987 \\
BPIC 1435 & Greece/Kavala & 1990 \\
ISPaVe 013 & Italy/Latium/Rome & 1991 \\
ISPaVe 011 & Italy/Latium/Rome & 1992 \\
ISPaVe 037 & Italy/Latium/Rome & 1993 \\
ISPaVe 439 & Italy/Latium/Rome & 1995 \\
ISPaVe 2056 & Italy/Latium/Viterbo & 1994 \\
ISPaVe 2057 & Italy/Latium/Viterbo & 1994 \\
ISPaVe 2058 & Italy/Latium/Viterbo & 1994 \\
ISPaVe 2059 & Italy/Latium/Viterbo & 1994 \\
ISPaVe 683 & Italy/Latium/Viterbo & 1996 \\
ISF H1 & Italy/Latium/Viterbo & 2002 \\
ISF H2 & Italy/Latium/Viterbo & 2003 \\
ISF H3 & Italy/Latium/Viterbo & 2003 \\
ISF H4 & Italy/Latium/Viterbo & 2004 \\
& & \\
\hline
\end{tabular}

${ }^{*}$ All strains were isolated from diseased tissue and confirmed to be pathogenic on hazelnut.

correction $\left(\alpha=0.2\right.$, obtained from PAUP $\left.^{*}\right)$. The neighbour-joining trees were bootstrapped with 1000 pseudo-replicates, and rooted with orthologous sequences from Pseudomonas fluorescens Pf0-1 (US Department of Energy, Joint Genome Institute), although this sequence is not presented in Fig. 1 to improve clarity. The maximum-likelihood tree was midpoint rooted.

The Shimodaira-Hasegawa (SH) test (Shimodaira \& Hasegawa, 1999) was used to test phylogenetic congruence between maximumlikelihood gene trees. The SH test determines the likelihood of a dataset given alternative trees. This analysis was performed with the DNAML module of PHYLIP. Genetic distances were calculated in MEGA3 using a Kimura two-parameter model with gamma correction

Table 2. Genomic position of MLST loci

Relative position of loci along the chromosome. Numbers are in $\mathrm{kb}$ relative to the origin of replication. $P$. syringae has a genome size of $\sim 6.5 \mathrm{Mb}$.

\begin{tabular}{|lcc|}
\hline Locus & Phylogroup 1 $\dagger$ & Phylogroup 2 $\ddagger$ \\
\hline gapA & 1415929 & 1255529 \\
gltA & 2415034 & 2325223 \\
gyrB & 4544 & 4206 \\
rpoD & 590198 & 5502717 \\
\hline
\end{tabular}

*The phylogroup 1 strains have a rearrangement that encompasses only the genomic region that includes $r p o D$.

$\dagger$ Based on the sequenced genome of $P$. syringae pv. tomato DC3000. $\ddagger$ Based on the sequenced genome of $P$. syringae pv. syringae B728a. 


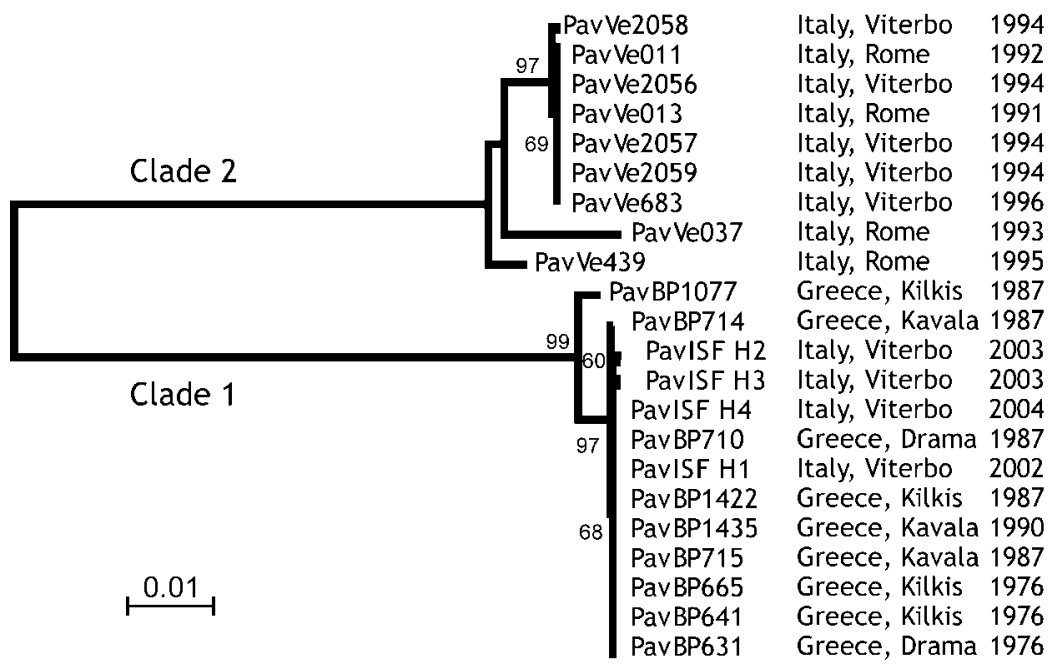

Fig. 1. Neighbour-joining tree of Pav isolates from Greece and Italy. Bootstrap confidence values $\geqslant 60$ are presented at each node. See Methods for details of tree construction. The location and year of isolation are presented to the right of each strain name. Clades 1 and 2 correspond to $P$. syringae phylogroups 1 and 2, respectively (see Fig. 2).

$(\alpha=0.2)$. Population genetic statistics were calculated in DnaSP ver4.10.7 (Rozas et al., 2003).

\section{RESULTS}

Twenty-two Pav strains were typed at four loci for MLST analysis, gapA, gltA, gyrB and $r p o D$. Each locus provided between 494 and 529 bp of common sequence, resulting in a total sequence length of $2040 \mathrm{bp}$. These data were integrated into the overall $P$. syringae MLST database (Hwang et al., 2005; Sarkar \& Guttman, 2004) for some of the phylogenetic analyses. Independent phylogenetic analyses were performed on the four loci in addition to a combined analysis on the concatenated sequence datasets.

Two distinct clades were observed in all four sequenced genes (Fig. 1). The clade $1 \mathrm{Pav}$ isolates were collected from the original 1976 epidemic in northern Greece (Drama and Kilkis), during the late 1980s and early 1990s in the Kilkis, Kavala and Drama regions of Greece, and between 2002 and 2004 in Viterbo, Italy. These strains are highly homogeneous, with the only significant polymorphism found in the 1987 Greek isolate PavBP1077 from Kilkis. The clade 2 Pav strains were all Italian isolates from either Viterbo or Rome collected from 1991 to 1995 . Most of these strains are extremely homogeneous, with the only exceptions being PavVe037 and PavVe439, which were collected in Rome in 1993 and 1995, respectively.

A Shimodaira-Hasegawa $(\mathrm{SH})$ test was performed on the maximum-likelihood (ML) gene trees from each locus to determine if the gene trees were congruent enough to permit the data to be combined (Table 3). The gapA and $g l t A$ sequences are compatible with the ML trees generated from all of the loci, while the $g y r B$ and $r p o D$ sequences were not compatible with the trees generated from gapA and gltA. The sequence data from all of the loci were compatible with the ML tree generated from the combined
Table 3. Shimodaira-Hazegawa (SH) analysis of tree congruence

\begin{tabular}{|c|c|c|c|c|c|}
\hline Data & Tree & $\log L^{*}$ & $\begin{array}{c}\text { Diff. } \\
\log L \dagger\end{array}$ & $P \ddagger$ & $\begin{array}{c}\text { Signif. } \\
\text { worse?\$ }\end{array}$ \\
\hline \multirow[t]{5}{*}{$g a p A$} & gapA & -943.5 & $\leftarrow$ best & & \\
\hline & gltA & -945.0 & -1.5 & 0.284 & No \\
\hline & gyrB & -946.2 & -2.6 & 0.142 & No \\
\hline & $r p o D$ & -946.2 & -2.6 & 0.142 & No \\
\hline & Combined & -946.2 & -2.6 & 0.142 & No \\
\hline \multirow[t]{5}{*}{ gltA } & gapA & -990.7 & -1.9 & 0.320 & No \\
\hline & gltA & -988.7 & $\leftarrow$ best & & \\
\hline & $\operatorname{gyr} B$ & -990.7 & -1.9 & 0.321 & No \\
\hline & $r p o D$ & -990.7 & -1.9 & 0.321 & No \\
\hline & Combined & -990.7 & -1.9 & 0.321 & No \\
\hline \multirow[t]{5}{*}{ gyrB } & gapA & -1087.9 & -43.4 & 0.011 & Yes \\
\hline & gltA & -1086.2 & -41.7 & 0.007 & Yes \\
\hline & gyr $B$ & -1044.5 & $\leftarrow$ best & & \\
\hline & $r p o D$ & -1051.1 & -6.6 & 0.395 & No \\
\hline & Combined & -1044.5 & 0.0 & 0.866 & No \\
\hline \multirow[t]{5}{*}{$r p o D$} & gapA & -994.5 & -28.0 & 0.025 & Yes \\
\hline & gltA & -997.8 & -31.3 & 0.013 & Yes \\
\hline & $\operatorname{gyr} B$ & -966.5 & 0.0 & 0.775 & No \\
\hline & $r p o D$ & -966.5 & $\leftarrow$ best & & \\
\hline & Combined & -966.5 & 0.0 & 0.999 & No \\
\hline \multirow[t]{5}{*}{ Combined } & gapA & -4036.7 & -66.0 & 0.003 & Yes \\
\hline & gltA & -4034.8 & -64.1 & 0.003 & Yes \\
\hline & $\operatorname{gyr} B$ & -3970.7 & 0.0 & 0.738 & No \\
\hline & $r p o D$ & -3980.0 & -9.3 & 0.419 & No \\
\hline & Combined & -3970.7 & $\leftarrow$ best & & \\
\hline
\end{tabular}

${ }^{\star}$ Log-likelihood score for the fit of the data given the tree.

$\dagger$ Difference between the observed log-likelihood of the data given the tree in question, and the log-likelihood of the data given the 'best' tree (the tree that results in the highest likelihood given the data). $\ddagger P$-value of significance for the difference in log-likelihood. $§$ Qualitative assessment of whether the specified tree is a significantly worse fit relative to the 'best' tree, given the data. 


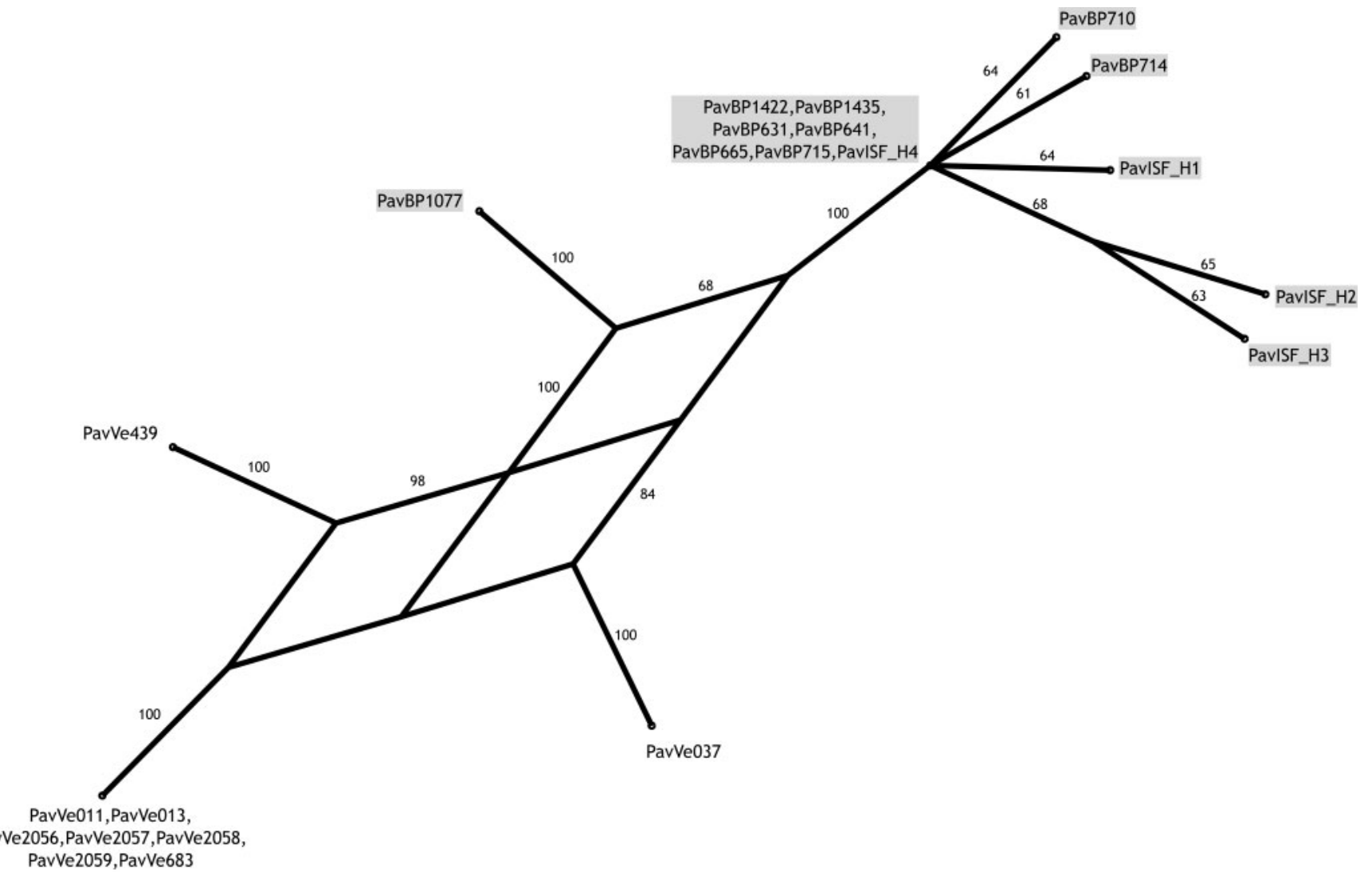

Fig. 2. Split decomposition analysis of $P a v$ isolates from Greece and Italy. Bootstrap confidence values are presented along each branch. Pav strains from phylogroup 1 are presented in shaded boxes, while Pav strains from phylogroup 2 are presented without shading. PavBP1077 is positioned within a network between the phylogroup 1 and phylogroup 2 strains, supporting past genetic exchange between these highly divergent groups.

dataset; consequently, we combined the data from the four loci for most of the further analyses.

The only significant inconsistency between the gapA and glt $A$ gene trees, and the gyrB and $r p o D$ gene trees, is with respect to the branching of Rome isolates $\mathrm{PavVe} 037$ and $\mathrm{PavVe} 439$ from clade 2. All four gene genealogies indicate that both of these strains diverged prior to the diversification of the rest of the clade 2 strains, but the gapA and gltA gene genealogies indicate that $\mathrm{PavVe} 439$ diverged prior to PavVe037, while the $g y r B$ and $r p o D$ gene genealogies indicate the reverse branching order.

A split decomposition analysis on the combined Pav dataset reveals a network structure connecting $\mathrm{PavVe} 037$ and PavVe439 from clade 2 and PavBP1077 from clade 1 (Fig. 2). PavBP1077 was isolated in Kilkis, Greece, in 1987 and is the most basal branching of the clade 1 strains. Although PavBP1077 is separated from the two clade 2 strains by a large genetic distance, these three strains show significant reticulation via a bootstrap test along all orthogonal edges of the network. These results support the action of recombination rather than homoplasy in generating the reticulation, and indicate that there has been genetic exchange between these genetically distinct clades.

The overall pairwise nucleotide diversity $(\pi)$ of all Pav strains for the combined dataset is 0.0465 (Table 4), with a

Table 4. Population genetic statistics

\begin{tabular}{|lrccc|}
\hline Category & $\boldsymbol{N}^{*}$ & $\begin{array}{c}\text { Seg. } \\
\text { sites } \dagger\end{array}$ & $\begin{array}{c}\text { No. of } \\
\text { haplotypes }\end{array}$ & $\boldsymbol{\pi} \ddagger$ \\
\hline Phylogroup (clade) 1 & 13 & 1 & 7 & 0.0015 \\
Phylogroup (clade) 2 & 9 & 48 & 4 & 0.0059 \\
Greek isolates & 9 & 15 & 4 & 0.0016 \\
Italian isolates & 13 & 207 & 8 & 0.0441 \\
Italian 1990s isolates & 9 & 48 & 4 & 0.0059 \\
Italian 2000s isolates & 4 & 4 & 4 & 0.0011 \\
Total & 22 & 213 & 11 & 0.0465 \\
\hline
\end{tabular}

${ }^{*}$ Number of strains in the specified category.

$\dagger$ Number of segregating sites.

$\ddagger$ Mean pairwise nucleotide diversity. 
genetic distance $(\mathrm{K} 2 \mathrm{P}-\Gamma)$ between the clade 1 and clade 2 strains of 0.066 and an Fst of 0.962 (Hudson et al., 1992). The pairwise nucleotide diversity within clades 1 and 2 is 0.0015 and 0.0059 , respectively, supporting strong genetic differentiation between the two clades. Clustering the strains by geographical origin shows that the Greek strains have a mean pairwise nucleotide diversity of only 0.0016 , while the diversity among the Italian strains is 0.0441, reflecting the fact that the Greek strains are restricted to clade 1, while Italian strains are found among both clades. There is no genetic structure among subregions within either Greece or Italy, but there is complete genetic separation between the Italian strains isolated during the 1990s and those isolated in the 2000s, with an Fst for these two epidemics of 0.964 .

Without any further data, the finding of two distinct Pav clades could simply be interpreted as divergence from an ancestral hazelnut pathogen. Alternatively, these results may indicate that the two lineages independently converged on hazelnut pathogenesis from different ancestral states. When the Pav data are integrated into the full $P$. syringae MLST dataset (Hwang et al., 2005; Sarkar \& Guttman, 2004), it is clear that hazelnut pathogens are a polyphyletic group (Fig. 3). In other words, hazelnut pathogenesis is a trait that has evolved independently at least twice in the history of $P$. syringae. The clade 1 Pav strains fall into $P$. syringae phylogenetic group (phylogroup) 1 , while the clade 2 Pav strains fall into phylogroup 2. Although phylogroup 1 is dominated by pathogens of tomato and brassicaceous crops, the Pav clade 1 strains are most closely related to the European plum (Prunus domestica L.) isolate $P$. syringae pv. morsprunorum 19322 (Pmp19322), the tea (Thea chinensis L.) pathogen $P$. syringae pv. theae K93001 (PthK93001) and the kiwifruit (Actinidia chinensis Ferg.) pathogen P. syringae pv. actinidiae FTRS_L1 (Pan FTRS_L1). The Pav clade 2 strains in phylogroup 2 are nested within a large and highly diverse clade with strains that infect a range of hosts, including corn, lilac, zucchini and pear. Within this group of strains are two distinct clusters of pea pathogens, including one cluster that branches off basally and is quite closely related to PavVe037. Consequently, it appears as if the Pav clade 2 strains may have diverged from an ancestral pea pathogen.

\section{DISCUSSION}

The polyphyly observed among these Pav isolates provides strong support for pathogenic convergence onto a common hazelnut host. The first appearance of bacterial canker of hazelnut occurred in Greece in 1976. The extreme degree of homogeneity between these original isolates and the bulk of the clade 1 strains indicates that this clone has successfully propagated and spread to multiple locations within Greece during the 1970s and 1980s and Italy during the 2000s. This last observation is particularly interesting since there has been no exchange of hazelnut material between Greece and Italy in recent years, raising the possibility of either illicit or unintentional human transport of propagative material or environmental movement via an unknown vector. The small degree of diversity found in the clade 1 strain PavBP1077 may be due to the movement of genetic material from the original hazelnut pathogen clonal group to a closely related strain. Alternatively, PavBP1077 may be divergent due to the movement of genetic material from a closely related strain into a member of the original hazelnut pathogen clonal group.

The hazelnut decline epidemic that occurred in Italy during the 1990s was clearly caused by strains of a different origin than the original Greek epidemic. The source of these strains is unclear, although, intriguingly, there is evidence of recombination between a strain in this clade and strains from the highly divergent phylogroup 1 Pav clade. The recombination seen in the phylogroup 1 strain PavBP1077 may have moved virulence factors between these divergent groups of $P$. syringae, thereby conferring the ability to infect hazelnut. Based on the phylogenetic position of the Pav clade 2 strains it is possible that the recipient strain was a pea (Pisum sativum L.) pathogen. This speculation is supported by the fact that crops from the Leguminosae family are traditionally grown in the Latium region of Italy where the hazelnut decline epidemic first appeared. In addition, plant species of this family are an important part of the local wild flora.

The finding that recombination has influenced the evolution of the Pav strains at the MLST housekeeping loci is unexpected given the very highly level of clonality observed within the P. syringae species complex (Hwang et al., 2005; Sarkar \& Guttman, 2004). Clonality in this context means that genetic variation is much more likely to be introduced through the mutational process than through the action of homologous recombination. Consequently, in highly clonal species, the vast majority of alleles share a common evolutionary history, and their divergence can be traced vertically back to a common ancestor. Recombination generally gives rise to gene genealogies inconsistent with vertical descent, or in the case of multilocus data, evolutionary networks rather than simple bifurcating trees. The $\mathrm{SH}$ tests show that the recombination event that occurred among the Pav strains influenced the gapA and gltA loci as a block relative to the grrB and $r p o D$ loci. Given the present data, it is difficult to conclusively determine the polarity of movement, or even which block of loci were affected. Nevertheless, it is interesting that, at least with respect to the sequenced genome of P. syringae pv. tomato DC3000 (a phylogroup 1 strain, GenBank accession AE016853), the region between the gapA and $g l t A$ loci includes the $h r p / h r c$ cluster encoding the type III secretion system and many of its effectors, while the region between $\operatorname{gyr} B$ and $r p o D$ includes the type III effectors hopF, hopS and hopK. The type III secretion system and its effectors are known to be important determinants of virulence and host specificity.

There are a number of alternative explanations for the evolution of hazelnut pathogenesis in two distinct lineages. 


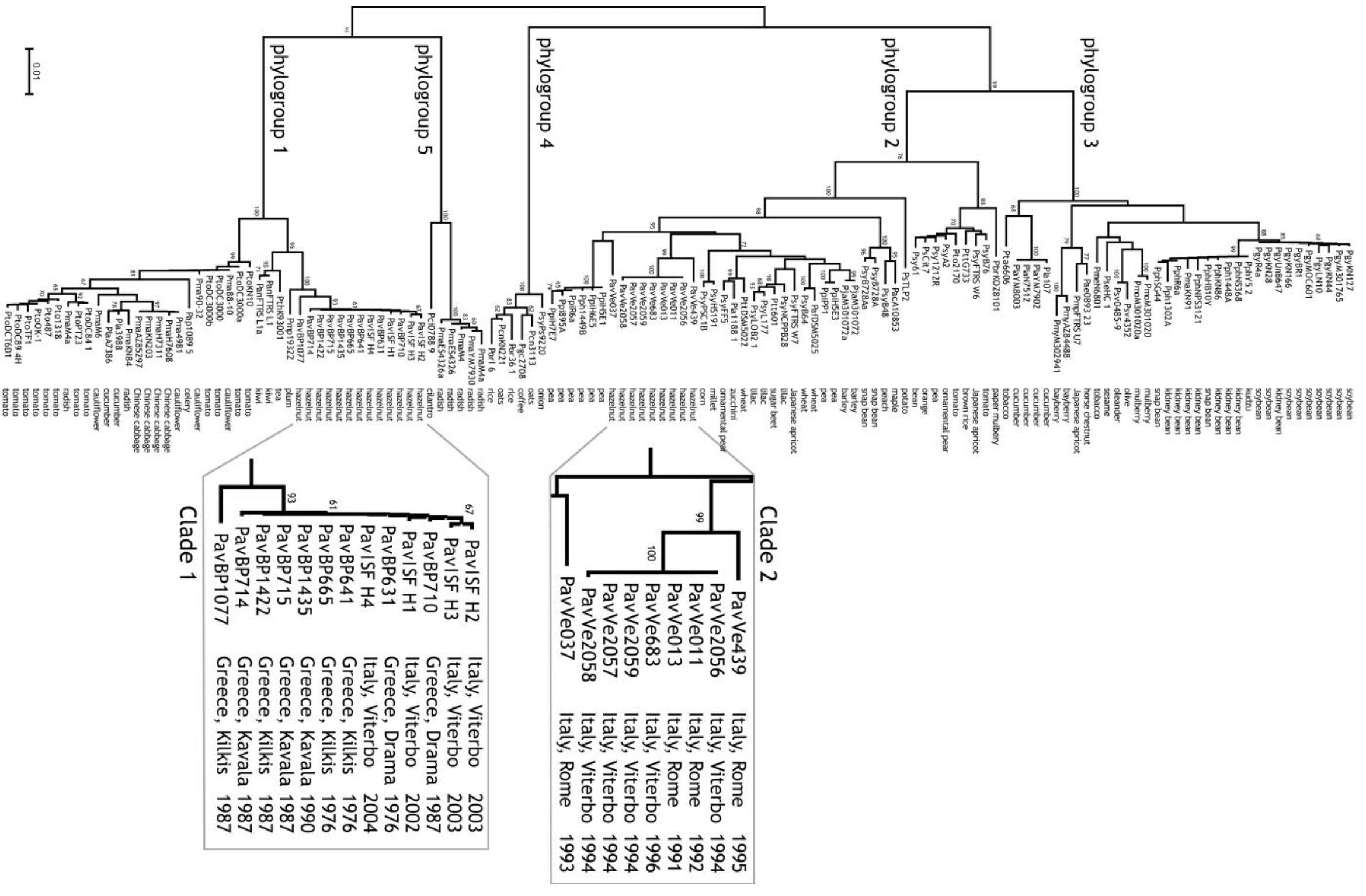

Fig. 3. Neighbour-joining tree of the $P$. syringae species complex based on four-locus MLST. The positions of the Pav strains are highlighted in the blowup boxes to the right of the tree, and annotated with the location and year of isolation. Phylogenetic robustness was evaluated by bootstrap analysis, with bootstrap confidence values $\geqslant 60$ presented at each node. The host of isolation for each strain is presented to the right of the tree. A complete strain list is available from the corresponding author on request. 
Ancestral strains could have independently acquired the necessary virulence factors from another source, or alternatively, both ancestral strains may have shared the necessary set, and independently lost factors that restrict growth on this host. The ability to cause disease on hazelnut may have an even simpler explanation, such as only requiring small mutational changes to key genes rather than larger-scale acquisition or loss of virulenceassociated genes. This latter explanation would imply that the two Pav lineages resulted from de novo local adaptation. The answer to these intriguing questions will require a much more thorough comparative examination of the host ranges and suites of virulence factors carried by the phylogroup 1 and 2 Pav strains. It will also be necessary to examine host range and profiles of virulence-associated genes in closely related strains that are non-pathogenic on hazelnut in order to polarize the evolutionary changes. While these studies are technically challenging, they will undoubtedly provide valuable information on the factors that are necessary for hazelnut host-specificity and pathogenesis, and on the evolution of host specificity and virulence in general.

Finally, there has been some disagreement as to the taxonomic distinctiveness of the Pav isolates (Janse et al., 1996). Some researchers have preferred to consider these strains as constituting a distinct species from $P$. syringae based on their host specificity and the fact that they do not grow on nutrient agar (Scortichini \& Angelucci, 1999). However, this MLST analysis clearly indicates that Pav strains are not only polyphyletic, but convincingly embedded within major $P$. syringae phylogroups. This study supports the maintenance of $\mathrm{Pav}$ as a pathovar within the $P$. syringae species complex.

\section{ACKNOWLEDGEMENTS}

D. S. G. is supported by grants from the Canada Research Chairs program, the National Sciences and Engineering Research Council of Canada, and Performance Plants Inc. of Kingston ON.

\section{REFERENCES}

Chenna, R., Sugawara, H., Koike, T., Lopez, R., Gibson, T. J., Higgins, D. G. \& Thompson, J. D. (2003). Multiple sequence alignment with the Clustal series of programs. Nucleic Acids Res 31, 3497-3500.

Felsenstein, J. (1993). PHYLIP (Phylogeny Inference Package). Distributed by author. Department of Genetics, University of Washington, Seattle, USA.

Hudson, R. R., Slatkin, M. \& Maddison, W. P. (1992). Estimation of levels of gene flow from DNA sequence data. Genetics 132, 583-589.
Hwang, M. S. H., Morgan, R. L., Sarkar, S. F., Wang, P. W. \& Guttman, D. S. (2005). Phylogenetic characterization of virulence and resistance phenotypes in Pseudomonas syringae. Appl Environ Microbiol 71, 5182-5191.

Janse, J. D., Rossi, P., Angelucci, L., Scortichini, M., Derks, J. H. J., Akkermans, A. D. L., DeVrijer, R. \& Psallidas, P. G. (1996). Reclassification of Pseudomonas syringae pv avellanae as Pseudomonas avellanae (spec nov), the bacterium causing canker of hazelnut (Corylus avellana L). Syst Appl Microbiol 19, 589-595.

King, E. O., Ward, M. K. \& Raney, D. E. (1954). Two simple media for the demonstration of phycocyanin and fluorescin. J Lab Clin Med 44, 301-307.

Kumar, S., Tamura, K. \& Nei, M. (2004). MEGA3: integrated software for Molecular Evolutionary Genetics Analysis and sequence alignment. Brief Bioinform 5, 150-163.

Ovod, V. V., Knirel, Y. A., Samson, R. \& Krohn, K. J. (1999). Immunochemical characterization and taxonomic evaluation of the $O$ polysaccharides of the lipopolysaccharides of Pseudomonas syringae serogroup O1 strains. J Bacteriol 181, 6937-6947.

Psallidas, P. G. \& Panagopoulos, C. G. (1979). A bacterial canker of hazelnut in Greece caused by Pseudomonas syringae pv. avellanae. J Phytopathol 94, 103-111.

Rozas, J., Sanchez-DelBarrio, J. C., Messeguer, X. \& Rozas, R. (2003). DnaSP, DNA polymorphism analyses by the coalescent and other methods. Bioinformatics 19, 2496-2497.

Sarkar, S. F. \& Guttman, D. S. (2004). The evolution of the core genome of Pseudomonas syringae, a highly clonal, endemic plant pathogen. Appl Environ Microbiol 70, 1999-2012.

Scortichini, M. (2002). Bacterial canker and decline of European Hazelnut. Plant Dis 86, 704-709.

Scortichini, M. \& Angelucci, L. (1999). Phenotypic characterization of Pseudomonas avellanae (Psallidas) Janse et al. and occurrence of colony variants. J Plant Pathol 81, 55-61.

Scortichini, M., Dettori, M. T., Marchesi, U., Palombi, M. A. \& Rossi, M. P. (1998). Differentiation of Pseudomonas avellanae strains from Greece and Italy by rep-PCR genomic fingerprinting. J Phytopathol 146, 417-420.

Scortichini, M., Marchesi, U., Rossi, M. P. \& Di Prospero, P. (2002). Bacteria associated with hazelnut (Corylus avellana L.) decline are of two groups: Pseudomonas avellanae and strains resembling P. syringae pv. syringae. Appl Environ Microbiol 68, 476-484.

Scortichini, M., Natalini, E. \& Angelucci, L. (2003). Clonal population structure of Pseudomonas avellanae strains of different origin based on multilocus enzyme electrophoresis. Microbiology 149, 2891-2900.

Scortichini, M., Rossi, M. P., Loreti, S., Bosco, A., Fiori, M., Jackson, R. W., Stead, D. E., Aspin, A., Marchesi, U. \& other authors (2005). Pseudomonas syringae pv. coryli, the causal agent of bacterial twig dieback of Corylus avellana. Phytopathology 95, 1316-1324.

Shimodaira, H. \& Hasegawa, M. (1999). Multiple comparisons of log-likelihoods with applications to phylogenetic inference. Mol Biol Evol 16, 1114-1116.

Swofford, D. L. (1993). PAUP: Phylogenetic Analysis Using Parsimony. Champaign, IL: Illinois Natural History Survey.

Edited by: Fred A. Rainey 